\title{
FARMACI A PICCOLE DOSI: LA SCIENZA MEDICA NEI PRIMI DIZIONARI INGLESI
}

\author{
Nota del s.c. GIOVANNI IAMARTINO (*)
}

(Adunanza del 22 marzo 2018)

SunTO. - La nota prende in esame il ruolo della lessicografia nella divulgazione della terminologia medico-scientifica nell'Inghilterra del Seicento. Di fatto, la compilazione dei primi dizionari inglesi monolingui procedette parallelamente alla nascita e ai primi sviluppi della scienza moderna. Dopo aver tratteggiato la tradizione lessicografica dei cosiddetti hard-word dictionaries e aver mostrato come queste opere vennero coinvolte nella divulgazione della scienza, e soprattutto della terminologia scientifica, nel Seicento, la nota si focalizza sulle voci mediche nel primissimo dizionario inglese monolingue, $A$ table alphabeticall di Robert Cawdrey, pubblicato nel 1604.

ABSTRACT. - This essay examines the role of lexicography in the popularisation of medical terminology in seventeenth-century England. As a matter of fact, the compilation of the earliest monolingual English dictionaries proceeded side by side with the birth and early development of modern science. After sketching the lexicographical tradition of the so-called hard-word dictionaries and commenting on how these works were involved in the popularisation of science, and especially scientific terminology, in the seventeenth century, this essay focuses on all the medical entries in the very first monolingual English dictionary, Robert Cawdrey's A table alphabeticall of 1604.

\section{INTRODUZIONE}

Nell'Europa occidentale, i primi dizionari monolingui compaiono all'inizio del Seicento: si tratta di opere corpose quali il Thresor de la

(*) Istituto Lombardo Accademia di Scienze e Lettere, Milano; Università degli Studi di Milano, Italy. E-mail: giovanni.iamartino@unimi.it 
langue françois di Jean Nicot, il Tesoro de la lengva castellana, o española di Sebastián de Covarrubias, e il Vocabolario degli Accademici della Crvsca. ${ }^{1}$ Queste sono precedute da uno smilzo libretto intitolato $A$ table alphabeticall che inaugura, nel 1604, la tradizione della lessicografia inglese monolingue inglese. ${ }^{2}$ Come altrove in Europa, anche in Inghilterra la produzione di dizionari monolingui è preceduta da quella di dizionari bilingui e plurilingui; ${ }^{3}$ ma diversamente dal Continente tale produzione si avvia e si sviluppa attraverso una sequenza di cosiddetti hard-word dictionaries, dizionari delle parole difficili, il cui scopo

was to transfer the whole current Latin vocabulary, already understood by scholars, into English. Such dictionaries were for the educationally insecure: non-scholars, the nouveau riche aspirants to a higher class merchants, artisans, and the like - and the wives of already established gentry. Thus their dictionaries were heavily infused with Latin-derived terms. Above all their publication was intended to broaden the basis of the educated élite; the illiterate masses remained irrelevant. ${ }^{4}$

1 J. Nicot, Thresor de la langue françoyse tante ancienne que moderne, Paris, David Douceur, 1606; S. de Covarrubias, Tesoro de la lengva castellana, o española, Madrid, Luis Sanchez, 1611; Vocabolario degli Accademici della Crusca, Venezia, Giouanni Alberti, 1621.

2 R. Cawdrey, A table alphabeticall ..., London, Edmund Weauer, 1604. Questo dizionario, che sopravvive in un'unica copia conservata nella Bodleian Library di Oxford, può essere facilmente consultato nella collana EEBO - Early English Books Online. E' pure disponibile un'edizione in facsimile a cura di J. Simpson, Oxford, Bodleian Library, 2007.

Ai fini della discussione che segue merita riprodurre nella sua interezza il frontespizio di questo dizionario, il cui autore, Robert Cawdrey, compare come firmatario della dedica del dizionario: «A table alphabeticall, conteyning and teaching the true vvriting, and vnderstanding of hard vsuall English wordes, borrowed from the Hebrew, Greeke, Latine, or French. \&c. With the interpretation thereof by plaine English words, gathered for the benefit \& helpe of Ladies, Gentlewomen, or any other vnskilfull persons. Whereby they may the more easilie and better vnderstand many hard English words, which they shall heare or read in Scriptures, Sermons, or elswhere, and also be made able to vse the same aptly themselues. Legere, et non intelligere, neglegere est. As good not read, as not to vnderstand. At London, printed by I. R. for Edmund Weauer, \& are to be sold at his shop at the great north doore of Paules Church. 1604».

3 Per una presentazione panoramica su questo tema, si veda G. Stein, The English dictionary before Cawdrey, Tübingen, Niemeyer, 1985.

4 J. Green, Chasing the sun: Dictionary-makers and the dictionaries they made, London, Jonathan Cape, 1996, 147. Una bibliografia essenziale sulla storia della lessicografia inglese comprende: D.T. Starnes e G.E. Noyes, The English dictionary from 
Queste considerazioni - la trasmissione del sapere dai dotti alle persone meno istruite, e la sfida del vernacolo al predominio del latino come lingua della cultura - si ritrovano sussunte nel frontespizio del dizionario di Cawdrey (citato nella nota 2 ) dove si specifica che le «hard vsuall English words», ossia la terminologia dei diversi ambiti scientifici e culturali, viene spiegata nel dizionario mediante «plaine English words, gathered for the benefit \& helpe of Ladies, Gentlewomen, or any other vnskilfull persons». Si noti che Cawdrey non è, non vuole essere politicamente scorretto qui: dame e gentildonne fanno comunque parte degli strati sociali privilegiati; hanno mezzi e istruzione (altrimenti non sarebbero in grado di acquistare e consultare il dizionario), ma sono «vnskilfull» perché, in quanto donne, non hanno accesso agli studi universitari; dunque, non possono apprendere le lingue classiche e impadronirsi delle conoscenze specialistiche veicolate da tali lingue. Di conseguenza, sono disarmate di fronte alle terminologie delle diverse discipline, che il frontespizio dichiara essere «borrowed from the Hebrew, Greeke, Latin, or French. \&c.», ma sono per la maggior parte di derivazione latina. Infatti, nelle ultime righe della prefazione «To the Reader», Cawdrey spiega che

whereas all such words as are deriued \& drawne from the Greek, are noted with these $[$ sic $]$ letter, $(\mathrm{g})$. And the French are marked thus $(\mathbb{S})$ but such words as are deriued from the latin, haue no marke at all.

- così chiarendo che la maggior parte dei tecnicismi nell'inglese della prima età moderna, a qualunque campo semantico o dominio concettuale si riferiscano, sono di derivazione latina, e come tali non marcati nel dizionario.

Cawdrey to Johnson 1604-1755, nuova ed. a cura di G. Stein, Amsterdam Philadelphia, Benjamins, 1991; T. Hayashi, The theory of English lexicography 15301791, Amsterdam, Benjamins, 1978; J. Schäfer, Early modern English lexicography, Oxford, Clarendon Press, 1989; Ph. Benson, Ethnocentrism and the English dictionary, London - New York, Routledge, 2001, 61-82; Words and dictionaries from the British isles in historical perspective, a cura di J. Considine e G. Iamartino, Newcastle upon Tyne, Cambridge Scholars Publishing, 2007; The Oxford history of English lexicography, a cura di A.P. Cowie, 2 voll., Oxford, Clarendon Press, 2009; H. Béjoint, The lexicography of English, Oxford, Oxford University Press, 2010, 50-95; K. Miyoshi, The first century of English monolingual lexicography, Newcastle upon Tyne, Cambridge Scholars Publishing, 2017. 
La spiegazione dei tecnicismi, e la connessa divulgazione del pensiero scientifico, vanno pertanto considerati tratti distintivi della nascente lessicografia inglese monolingue, i cui progressi nel Seicento corrono paralleli alla nascita e allo sviluppo della scienza moderna in Gran Bretagna, che col tempo venne ad abbandonare il latino e a promuovere - vuoi attraverso le traduzioni, vuoi con la pubblicazione di opere tecnico-scientifiche direttamente nella lingua nazionale - l'uso dell'inglese, così rivendicando la sua adeguatezza, sia lessicale sia stilistica, a trattare il discorso scientifico. ${ }^{5}$

Dato tale contesto storico-culturale, ci si propone qui di analizzare la terminologia medica, così come viene elencata e spiegata nella Table alphabeticall di Robert Cawdrey. Diversi sono i motivi che rendono opportuno focalizzare l'attenzione sulla terminologia medica: in primo luogo perché, storicamente, tale terminologia è il risultato dell'interazione fra la scienza medica e la medicina popolare e tradizionale; secondariamente, perché il discorso medico-scientifico, nella prima età moderna, suscita notevole interesse fra le persone istruite, non è limitato alla ristretta cerchia dei medici; infine, perché a quei tempi non esiste ancora la figura del lessicografo di professione, e più di un dizionario viene compilato proprio da medici. In un certo qual modo, dunque, la

5 Lo sfondo storico-culturale a cui si fa qui riferimento è ben delineato nelle seguenti opere: M. Hunter, Science and society in Restoration England, Cambridge, Cambridge University Press, 1981; H.J. Cook, The decline of the old medical regime in Stuart London, Ithaca-London, Cornell University Press, 1986; The medical revolution of the seventeenth century, a cura di R. French e A. Wear, Cambridge, Cambridge University Press, 1989. Ottime introduzioni al discorso medico-scientifico in lingua inglese si trovano in due volumi, entrambi a cura di I. Taavitsainen e P. Pahta: Early modern English medical texts, Amsterdam - Philadelphia, Benjamins, 2010 e Medical writing in early modern English, Cambridge, Cambridge University Press, 2011. Ovviamente, momento cardine dello sviluppo della scienza moderna in Inghilterra è la fondazione della Royal Society e la pubblicazione delle sue Philosophical Transactions negli anni immediatamente successivi al ritorno degli Stuart sul trono inglese nel 1660. Esempio paradigmatico del passaggio dal latino all'inglese come lingua della scienza può essere senz'altro il trattato di William Harvey sulla circolazione del sangue: pubblicato per la prima volta in latino a Francoforte nel 1628, e dunque evidentemente indirizzato alla comunità scientifica internazionale, viene tradotto in inglese e pubblicato a Londra nel 1653, per una diversa platea di destinatari: si veda su questo G. Iamartino, Translators as wordsmiths: Lexical innovations in Harvey's De motu cordis in English, in English words in time, a cura di M. Sturiale et al., Monza, Polimetrica, 2014, 59-78. 
ricerca sulla tradizione lessicografica può contribuire alla storia della divulgazione scientifica nell'Europa della prima età moderna ${ }^{6}$

\section{LeSSiCOGRAFiA INGLESE MONOLINGUE DEL SEICENTO}

All'analisi della terminologia medica in A table alphabeticall è necessario premettere alcune considerazione che riguardano la lessicografia del Seicento inglese, in modo da inquadrare le peculiarità del lavoro di Robert Cawdrey in un contesto che tali peculiarità possa spiegare e ricondurre a una tradizione di cui lo stesso Cawdrey è elemento propulsore.

Già si è detto che la sua compilazione avvia la produzione dei cosiddetti hard-word dictionaries: a quella di Cawdrey fanno seguito le opere di John Bullokar, Henry Cockeram, Thomas Blount, Edward Phillips e Elisha Coles, tutte pubblicate per la prima volta fra il 1616 e il 1677, e tutte (come del resto il dizionario di Cawdrey) ripubblicate più di una volta. ${ }^{7}$ Questa produzione precede - e, attraverso le riedizioni, accompagna - la compilazione, nel secolo successivo, sia dei cosiddetti universal o general purpose dictionaries, ovvero i dizionari che si propongono (almeno teoricamente) di raccogliere tutte le parole di una data lingua, sia dei dizionari enciclopedici e delle vere e proprie enciclopedie, tipici artefatti culturali del Settecento. Le informazioni che si possono trarre dal paratesto di questi dizionari seicenteschi dimostrano fino a qual punto, e quanto deliberatamente, i loro compilatori contribuirono alla divulgazione del discorso scientifico nella mutevole realtà socioculturale dell'Inghilterra del tempo.

Per quanto riguarda più specificatamente il sapere medico, si potrebbe argomentare che non fosse necessario divulgare tali conoscenze nell'Inghilterra della prima età moderna perché la medicina popolare

6 Un testo utile per ripercorrere alcuni momenti chiave della storia della divulgazione dei saperi scientifici in Inghilterra, vista soprattutto attraverso l'analisi linguistica, è The popularization of specialized discourse and knowledge across communities and cultures, a cura di S. Kermas e Th. Christiansen, Bari, Edipuglia, 2013.

7 J. Bullokar, An English Expositor, London, I. Legatt, 1616; H. Cockeram, An English Dictionarie, London, N. Butter, 1623; Th. Blount, Glossographia, London, H. Moseley, 1656; E. Phillips, The New World of English Words, London, N. Brooke, 1658; E. Coles, An English Dictionary, London, P. Parker,1677. 
era ampiamente praticata, di fatto da lungo tempo. Ma questo è esattamente il motivo per cui la divulgazione dei nuovi saperi era importante: se è pur vero che col tempo si imposero nuovi modelli di pensiero e nuove metodologie fondate sull'osservazione e l'interpretazione dei fenomeni fisici, la teoria degli umori e la tradizione ippocratico-galenica non mancarono di esercitare il loro influsso fin dentro il diciassettesimo secolo. ${ }^{8}$ Il nuovo approccio scientifico alla realtà ebbe un impatto diretto sul linguaggio, come è dimostrato dai seguenti tre fattori: l'impiego progressivamente più ampio dell'inglese, al posto del latino, come lingua della scienza; la conseguente introduzione in inglese di neologismi scientifici; e la promozione del cosiddetto plain style - uno stile semplice e depurato degli orpelli retorici - nella comunicazione dei saperi scientifici. ${ }^{9}$

I primi dizionari inglesi monolingui furono coinvolti da tali processi evolutivi in più di un modo. Innanzitutto, la stessa tradizione lessicografica degli hard-word dictionaries ci mostra che tali dizionari vennero compilati e pubblicati proprio allo scopo di spiegare la nuova terminologia tecnico-scientifica inglese. Secondariamente, la produzione di questi dizionari - che spesso si rifacevano, per il lemmario e le definizioni, ai precedenti dizionari bilingui latino-inglese - seguiva immediatamente o accompagnava la tendenza, tipica della prima età moderna, a fare propri e svelare i 'segreti' della scienza antica traducendo in inglese testi latini e greci, e poi divulgare nuove idee mediante la produzione scientifica in lingua inglese. Infine, la promozione del plain style e una facile compren-

8 Nell'amplissima bibliografia sull'evoluzione del pensiero medico-scientifico e la sua divulgazione in età moderna si possono ricordare, per la particolare attenzione al mondo inglese, le seguenti opere: A. Grafton, A. Shelford e N. Siriasi, New worlds, ancient texts: the power of tradition and the shock of discovery, Cambridge, MA London, The Belknap Press of Harvard University Press, 1992; The popularization of medicine 1650-1850, a cura di R. Porter, London - New York, Routledge, 1992; R. Porter, Disease, medicine and society in England, 1550-1860, Basingstoke - London, Macmillan, 1993; A. Wear, Knowledge and practice in English medicine, 1550-1680, Cambridge, Cambridge University Press, 2000; R. French, Ancient and moderns in the medical sciences. From Hippocrates to Harvey, Aldershot - Burlington, Ashgate, 2000; E.L. Furdell, Publishing and medicine in early modern England, Rochester, NY, University of Rochester Press, 2002.

9 Una sintetica presentazione di questi aspetti si trova in M. Gotti, Medical discourse: developments, $16^{\text {th }}$ and $17^{\text {th }}$ centuries, in Encyclopedia of language and linguistics, a cura di K. Brown, 2a ed., Oxford, Elsevier Science, vol. 7, 674-681. 
sione come scopo ultimo della comunicazione scritta e parlata informa il lavoro di questi lessicografici seicenteschi, come ben mostra $A$ table alphabeticall. A parte quanto dichiarato sul frontespizio, nella prefazione «To the Reader», Cawdrey descrive gli utenti ideali del suo dizionario come coloro i quali «haue occasion to speak publiquely before the ignorant people», e in quanto tali dovrebbero «labour to speake so as is commonly receiued, and so as the most ignorant may well vnderstand them». Se è di primaria importanza il farsi capire ( $«$ Do we not speak, because we would haue other to vnderstand vs?»), è opportune fare uso del «plainest \& best kind of speech» e di «such words as are commonlie receiued». Tutto ciò a beneficio di chi ascolta, ma anche di chi legge, come suggerisce il motto - in latino ma opportunamente anche tradotto in inglese inserito nel frontespizio: «Legere, et non intelligere, neglegere est. As good not read, as not to vnderstand».

Una prova della stretta connessione fra discorso scientifico e lessicografia nel Seicento inglese è costituita dalla menzione delle diverse arti e scienze nei paratesti dei dizionari, mediante una sorta di lenta progressione. Infatti, il frontespizio di $A$ table alphabeticall, come si è visto, concentra l'interesse su «hard vsuall English wordes», ma né la dedica né la prefazione «To the Reader» contengono alcun riferimento alla scienza. Parimenti, il frontespizio di An English Expositor proclama che il dizionario insegnerà «the interpretation of the hardest words vsed in our Language», ma John Bullokar, nella prefazione «To the Courteous Reader», annuncia l'inclusione nel dizionario di «diuers termes of art, proper to the learned in Logicke, Philosophy, Law, Physicke. Astronomie, \&c. yea, and Diuinitie it selfe, best knowen to the seuerall professors thereof». Henry Cockeram, il primo a definire la propria compilazione monolingue con la parola Dictionarie, si limita a usare l'espressione «hard English Words» nel frontespizio, anche se il suo dizionario, che è tripartito, include una sezione enciclopedica che estende lo scopo didattico e l'utilità del suo lavoro. Un elenco delle scienze e arti i cui termini sono spiegati nel dizionario compare per la prima volta sul frontespizio della Glossographia di Thomas Blount (1656): «the Terms of Divinity, Law, Physick, Mathematicks, Heraldry, Anatomy, War, Musick, Architecture; and of several other Arts and Sciences Explicated». Tale elenco è surclassato, per la quantità e la varietà degli ambiti tecnico-scientifici, da quello che Edward Phillips propone, coerentemente con l'ambizioso titolo del suo dizionario, sul frontespizio di The new world of English words: 
All those Terms that relate to the Arts and Sciences; whether Theologie, Philosophy, Logick, Rhetorick, Grammer, Ethicks, Law, Natural History, Magick, Physick, Chirurgery, Anatomy, Chimistry, Botanicks, Mathematicks, Arithmetick, Geometry, Astronomy, Astrology, Chiromancy, Physiognomy, Navigation, Fortification, Dialling, Surveying, Musick, Perspective, Architecture, Heraldry, Curiosities, Mechanicks, Staticks, Merchandize, Jewelling, Painting, Graving, Husbandry, Horsemanship, Hawking, Hunting, Fishing, \&c.

Infine, il più pratico e modesto dizionarietto compilato da Elisha Coles ritorna a un più umile e breve elenco dei «difficult Terms that are used in Divinity, Husbandry, Physick, Phylosophy, Law, Navigation, Mathematicks, and other Arts and Sciences».

I paratesti dei diversi dizionari qui considerati forniscono pure delle informazioni sul ruolo dei lessicografi come trasmettitori e divulgatori delle conoscenze scientifiche. $\mathrm{Ci}$ si presentano tutti come autorevoli e affidabili esperti: sia Robert Cawdrey sia Elisha Coles dichiarano di essere «school-masters»; John Bullokar si qualifica sul frontespizio come «I. B. Doctor of Physicke» e a ciò fa seguire un motto in greco e un emblema che rimanda all'Università di Cambridge, doppia allusione alla sua formazione universitaria; Thomas Blount si definisce come «T.B. of the InnerTemple, Barrester»; Edward Phillips dichiara di aver lavorato sotto l'egida delle università di Cambridge e Oxford, e fa seguire al frontespizio un elenco di esperti - 34 nomi per 44 arti e scienze - che lo avrebbero assistito nella selezione e compilazione delle voci tecnico-scientifiche. ${ }^{10}$ Infine, il frontespizio di An English Dictionarie qualifica Henry Cockeram semplicemente come «H.C. Gent.»: questo non è un dettaglio insignificante, perché ci ricorda che a quel tempo la lessicografia era poco più che un passatempo per gentiluomini, ossia persone istruite ma non professionisti, e che a loro era affidata la divulgazione della scienza.

10 Starnes e Noyes, The English dictionary from Cawdrey to Johnson 1604-1755, 50-54, aprono la serie degli studiosi di storia della lessicografia inglese che puntano il dito contro i plagi operati da Phillips nei confronti dei dizionari precedenti, in particolare quello di Blount; un'interpretazione, questa, ampiamente corretta in anni recenti da J. Considine, In praise of Edward Phillips, Studia Linguistica Universitatis Iagellonicae Cracoviensis, 132 (2015), 211-228. E se anche l'elenco di esperti citati da Phillips non corrispondeva effettivamente a un reale contributo di tali studiosi ma era stato inserito per scopi primariamente promozionali, ciò dimostra quanto gli hard-word dictionaries potessero essere percepiti come strumenti di divulgazione della scienza. 
Più di una volta in questi dizionari del Seicento si evidenzia la distinzione fra il lessicografo istruito, il cui scopo è ampliare o rafforzare le conoscenze di chi ha un livello d'istruzione inferiore ma forse non lontano dal suo, e gli specialisti delle varie scienze. Ad esempio, nella prefazione «To the Courteous Reader», Bullokar si augura che «the severall professors» delle diverse scienze non se ne avranno a male se «I open the signification of such words, to the capacitie of the ignorant, whereby they may conceiue and vse them as well as those which haue bestowed long study in the languages». Thomas Blount poi, descrivendo i diversi tipi di tecnicismi elencati nella Glossographia, afferma chiaramente che la spiegazione dei termini legali è «necessary for every Gentleman of Estate» e non è destinata agli «studied Professors of that noble Science», così come la sua trattazione lessicografica della terminologia medica è «fit for the knowledge of many, who neither profess the study of Physick, Anatomy nor Chyrurgery». In sintesi, la finalità divulgativa di questi hard-word dictionaries si rivolge al lettore istruito, e non ai dotti esperti delle varie arti e scienze che, come sottolinea opportunamente Blount nel suo «To the Reader», hanno a disposizione dei testi specialisti, compresi glossari e dizionari tecnici: ad esempio, risale al 1607 The Interpreter di John Cowell, un fortunatissimo dizionario legale in lingua inglese; e sebbene dei glossari di termini medici fossero già disponibili in Inghilterra, negli anni tra il 1655 e il 1657, ossia perfettamente a scavalco della Glossographia di Blount, vengono pubblicate due opere intitolate $A$ /The Physical Dictionary, i primi dizionari medici inglesi. ${ }^{11}$

${ }^{11}$ Si vedano G. Iamartino e G. Rovelli, A Physical Dictionary of 1655: When translating medical science is not enough, Token: A Journal of English Linguistics, in corso di stampa, e Jukka Tyrkko, A Physical Dictionary (1657): The first English medical dictionary, in Selected proceedings of the 2008 symposium on New Approaches in English Historical Lexis (HEL-LEX 2), a cura di R. McConchie et al., Somerville, MA, Cascadilla Proceedings Project, 2009, 171-89. Per la storia della terminologia medica inglese e della sua presentazione in glossari e dizionari sono essenziali due volumi di R. McConchie: Lexicography and physicke. The record of sixteenth-century English medical terminology, Oxford, Clarendon Press, 1997, e Discovery in haste. English medical dictionaries and lexicographers 1547 to 1796, Berlin -Boston, de Gruyter, in corso di stampa. Pure utile Cowie, The Oxford history of English lexicography, a cura di A.P. Cowie, vol. 2, 77-82. 


\section{Il LeSSiCo DELla Medicina in A TABLE ALPHABETICALL DI ROBERT CAWDREY}

In tale contesto socio-culturale si colloca l'opera di Robert Cawdrey ${ }^{12}$ come primo dizionario inglese monolingue e il suo trattamento lessicografico delle voci che, come verrà chiarito nella successiva esemplificazione, si possono riferire all'ambito della medicina. Queste, su un totale di circa 2500, sono 123 , dunque poco meno del 5\%:

abortiue, adustion, agonie, aliment, amorte, anatomie, antidote, aphorisme, asperation, astrictiuelastringent, carnositie, catharre, chirurgion, choller, circumcise, clister, concoct, contagious, contaminate, contaminouse, contusion, copulation, cordiall, corporall, corps, corpulent, corrosiue, costiue, crassitude, crude, deambulation, debilitie, decoction, decrepite, diet, distended, dolor, dolorous, emerods, entrals, epilepsis, eunuch, excrement, expire, exulcerate, fecunditie, feeble, feuer, fluxe, fomentation, furious, gargarise, generation, genitalles, gibbocitie, gustation, heckticke, imbecilitie, impotent, incision, incurable, infection, infirmitie, inspire, instinct, languishing, lassitude, laxatiue, lethall, lethargie, ligament, lunatick, maffle, maladie, maniacque, matrixe, medicine, melancholie, mentall, mutilate, nerue, noysome, nutriment, obstruction, oppilation, orifice, ossicle, pellicles, pest, pestiferous, physiognomie, phisicke, phlebotomie, phrensie, pinguiditie, potion, pregnant, preseruatiue, putrefy, quartane, respiration, rheume, rubicunde, salubritie, sanguine, sanitie, scarifie, sensuall, sex, sperme, splene, spongeous, sterilitie, tertian, vapor, vehement, ventricle, vigour, vlcer, vlcerate, unguent, vocall, vpbraid.

La ricerca ${ }^{13}$ ha già acclarato che Cawdrey basa il suo lavoro di

12 Per essere precisi, si dovrebbe dire di Robert Cawdrey e del figlio Thomas, poiché Robert - nel dedicare $A$ table alphabeticall ad alcune nobildonne - specifica che il dizionario «long ago for the most part, was gathered by me, but lately augmented by my sonne Thomas, who now is Schoolemaister in London».

$13 \mathrm{Al}$ di là di quanto si trova nelle trattazioni di più ampio respiro sulla storia della lessicografia inglese, Cawdrey e $A$ table alphabeticall sono l'oggetto specifico dei seguenti studi: E.A. Irons, Sir Robert Cawdrie: Rector of South Luffenham, 1571-87, Annual report and transactions of the Rutland archeological and natural history society, 14 (1916), 23-33; De W.T. Starnes, English dictionaries of the seventeenth century, 
compilazione su due tipi di fonti. La prima è costituita dai glossari in manuali didattici per l'insegnamento dell'inglese, in particolare l'ortografia delle parole, scelta non sorprendente data la professione di Cawdrey come maestro: si tratta nello specifico di The English schoolemaister (1596) di Edmund Coote, un manualetto per insegnare agli scolari a leggere e scrivere in inglese; alle pagine 74-93 si trova un glossario di 1368 parole - derivate dal greco, dal latino o dal francese - di cui Coote propone la corretta grafia e, nella maggior parte dei casi, il significato mediante un equivalente, tratto dalla lingua comune, o una brevissima glossa. ${ }^{14}$ La gran parte di questo materiale lessicografico viene adottato con minimi cambiamenti da Cawdrey, come si evidenzia mediante il confronto di alcune delle oltre 50 voci d'argomento medico che passano da Coote a Cawdrey:

University of Texas studies in English, 17 (1937), 15-51; G.E. Noyes, The first English English dictionary, Cawdrey's Table alphabeticall, Modern language notes, 58, 8 (1943), 600-605; Starnes e Noyes, The English dictionary from Cawdrey to Johnson 1604-1755, 13-19; R.A. Peters, Robert Cawdrey and the first English dictionary, Journal of English linguistics, 2 (1968), 29-42; J. Schäfer, The hard-word dictionaries: A re-assessment, Leeds studies in English, n.s. 4 (1970), 31-48; J.A. Riddell, The beginning: English dictionaries of the first half of the seventeenth century, Leeds studies in English, n.s. 7 (1974), 117-151; J.A. Riddell, Some additional sources for early English dictionaries, Huntington library quarterly, 46 (1983), 223-235; R.W. McConchie, What were Robert Cawdrey's hard words? Learned terms and A table alphabeticall (1604), in 'As who say' - Many happy returns: Essays in honour of Saara Nevanlinna, a cura di P. Pahta et al., Helsinki, Helsinki University Press, 1992, 51-58; S. Brown, Women and the godly art of rhetoric: Robert Cawdrey's puritan dictionary, Studies in English literature, 1500-1900, 41, 1 (2001), 133-148; J. Bately, Cawdrey, Robert (b. 1537/8?, d. in or after 1604), Church of England clergyman and lexicographer, Oxford dictionary of national biography, 2004, http://www.oxforddnb.com; G. Stein, Lexicographical method and usage in Cawdrey's A table alphabeticall (1604), Studia neophilologica, 82 (2010), 163-177; K. Miyoshi, Cawdrey's Table alphabeticall (1604) reconsidered: Its driving force for early English lexicography, in Adventuring in dictionaries. New studies in the history of lexicography, a cura di J. Considine, Newcastle upon Tyne, Cambridge Scholars Publishing, 2010, 14-22.

14 E. Coote, The English schoole-maister, London, Ralph Jackson and Robert Dextar, 1596. Per un inquadramento su questo e simili testi, si veda T. Nevalainen, Norm and usage in seventeenth-century English, in Norm and usage in language bistory, 1600-1900: A sociolinguistic and comparative perspective, a cura di G. Rutten et al., Amsterdam - Philadelphia, Benjamins, 2014, 103-128. 
Coote - agonie g. heauie passion.

Cawdrey - agonie, (gr) heauie passion, anguish, griefe

Coote - choller gr. a humor causing anger.

Cawdrey - choller, (gr) a humor causing anger

Coote - debilitie weaknes.

Cawdrey - debilitie, weakenes, faintnes.

Coote - emerods k. disease.

Cawdrey - emerods, (k) of disease

Coote - infection corrupting.

Cawdrey - infection, corrupting

Coote - lethergie gr. k. drowsie disease

Cawdrey - lethargie, $(\mathrm{g})(\mathrm{k})$ a drowsie and forgetfull disease.

Coote - lunatick wanting his wits.

Cawdrey - lunatick, wanting his wits, at a certaine time of the age of the moone

Talvolta, dunque, Cawdrey riproduce alla lettera la voce di The English schoole-maister (choller, emerods, infection), che fa da modello anche per le indicazioni sull'origine etimologica e per la convenzione della sigla $(\mathrm{k})$ che sta per a kind of.${ }^{15}$ Invece, le integrazioni alle voci di Coote si possono spiegare come aggiunte autonome di Cawdrey - questo sembra essere il caso di debilitie - oppure come esemplificazioni del secondo tipo di fonti a cui si ispira Cawdrey, ossia la lessicografia bilingue, in particolare i dizionari latino-inglese e francese-inglese: scelta opportuna, in quanto questi bilingui lemmatizzano parole latine o parole francesi di immediata derivazione latina, le quali sono spesso alla base dei tecnicismi medico-scientifici inglesi. Risulta evidente che

15 Utilizzando la distinzione proposta dalla riflessione metalessicografica tra genus proximum e differentia specifica, si può dire che l'uso della sigla $(\mathrm{k})$ richiami il primo elemento definitorio ma non il secondo: ad esempio, emerods designa certamente una malattia (o, meglio, una condizione patologica), e questo è il genus, ma manca quanto marcherebbe la differentia di questa malattia da altre. Si può qui aggiungere che Cawdrey non preleva dal glossario di Coote tutti i termini di carattere medico lì elencati: un esempio è rappresentato da «carbuncle $\mathrm{k}$. disease or stone». 
Cawdrey integra la definizione di agonie proposta da Coote con quanto trova in A dictionarie French and English (1593) di Claudius Hollyband, la cui definizione di agonie è appunto «anguish, griefe»; nel caso di lethargie, Hollyband non è utile in quanto la definizione da lui proposta equivale a quella di Coote («léthargie, vne maladie, the litargie, a drowsie disease: f.»), mentre l'elemento aggiunto - l'aggettivo forgetfull - è probabilmente estrapolato dalla Bibliotheca scholastica (1589) di John Rider, che definisce il termine «sleepy, drousie, and forgetful sicknes, whereby reason and memory also perisheth». ${ }^{16}$

Nessuno di questi tre dizionari, tuttavia, può giustificare l'aggiunta di Cawdrey alla definizione che Coote dà di lunatick. Per questa bisogna ricorrere agli altri bilingui latino-inglese pubblicati in Inghilterra fin da prima della metà del Cinquecento: si tratta delle compilazioni di Sir Thomas Elyot, Thomas Cooper e Thomas Thomas, tutte pubblicate in diverse edizioni e tutte geneticamente correlate tra loro: ${ }^{17}$ lo si vede bene proprio nel caso di lunaticus, che viene definito come «madde or sycke» da Elyot e Cooper, e «madde or franticke» da Thomas, ma tutti i dizionari specificano che l'accesso di follia si verifica «at a certayne time of the moone», sintagma alla base dell'aggiunta di Cawdrey alla glossa proposta Coote.

Detto altrimenti, non è difficile trovare in $A$ table alphabeticall delle voci che risalgano senz'altro a questi bilingui latino-inglese, come ad esempio «epilepsis, (g) the falling sicknes» o «phlebotomie, (g) letting bloud»; ma la predilezione di Cawdrey per voci lessicografiche brevi o brevissime impedisce di individuare la fonte specifica tra i diversi bilingui latino-inglese: se è pur vero che The dictionary di Elyot elenca «epilepsia, the fallynge syckenes.» e «phlebotomia, bloodeletinge.», è altrettanto vero che tutti i dizionari successive includono le medesime espressioni in definizioni più articulate e di carattere enciclopedico.

16 C. Hollyband, A dictionarie French and English, London, Thomas Woodcock, 1593; J. Rider, Bibliotheca scholastica, London, Ioseph Barnes, 1589.

17 Th. Elyot, The dictionary, London, Thomas Berthelet, 1538, venne ripubblicato come Bibliotheca Eliotae nel 1542, e in successive riedizioni (1545, 1548, 1552 e 1559) curate da Th. Cooper, a sua volta autore di un Thesaurus linguae romanae \& britannicae (London, H. Wykes, 1565); gli ultimi decenni del Cinquecento, e oltre, sono dominati da Th. Thomas, Dictionarium linguae latinae et anglicanae, Cambridge, Th. Thomas, 1587, così che Cawdrey potrebbe aver fatto uso di questa edizione o una delle successive pubblicate negli anni 1589, 1593, 1594, 1596 o 1600. 
Nemmeno è di aiuto, nella ricerca del dizionario di riferimento per Cawdrey, la più estesa delle voci di $A$ table alphabeticall relative alla medicina, ossia «gargarise, to wash the mouth, and throate within, by stirring some liquor vp and downe in the mouth.»: infatti, tutti i bilingui latino-inglesi condividono con Cawdrey la prima parte della definizione, mentre la seconda è solo sua. Così, solo talvolta si possono fare ipotesi di una qualche consistenza circa la fonte lessicografica utilizzata da Cawdrey, ad esempio quando definisce «rheume, (g) or catarre, a distilling of humors from the head»: questa formulazione sembra escludere una derivazione dai dizionari di Elyot e Cooper, che reiterano la formula «rbeuma, a rewme or distillation of humours», mentre le varie edizioni di Thomas hanno «rheuma, A reume, a cattharre, a distillation of humours.», cioè includono il termine cattharre che caratterizza la definizione di Cawdrey, anche se l'aggiunta «from the head» pare essere stata decisa autonomamente da lui. ${ }^{18}$

Venendo ora a considerare le voci mediche di $A$ table alphabeticall indipendentemente dalla loro fonte, ossia ponendosi nella prospettiva di chi questo dizionarietto aveva e usava, si possono fare alcune considerazioni sull'efficacia delle tecniche definitorie adottate da Cawdrey per presentare la terminologia medica - tecniche che, in termini generali, non si discostano da quanto era comune trovare nei glossari e nei dizionari pluringui del tempo. Abbastanza numerosi sono innanzitutto i casi in cui il termine medico - che può riferirsi all'anatomia o alla fisiologia del corpo umano o ancora a qualche pratica curativa - è spiegato mediante un semplice equivalente tratto dalla lingua comune:

asperation, breathing; clister, medicine; feuer, ague; genitalles, priuities; infection, corrupting; lassitude, wearines; $\$$ maladie, disease; matrixe, wombe; nerue, sinewe; nutriment, nourishment; orifice, mouth; ossicle, bone; pellicles, skinnes; phrensie, (g) madnes; potion, a drinke; respiration, breathing out; sperme, seede; splene, milt.

$\mathrm{Al}$ di là delle eventuali incertezze ortografiche - asperation era più correttamente aspiration in Coote - si può notare come l'equivalente

18 E' meno rilevante il fatto che Cawdrey usi distilling anziché distillation perché le due forme sono presentate come varianti in A table alphabeticall e, unite da una parentesi graffa, vengono congiuntamente definite con «dropping downe by little and little». 
proposto sia talvolta impreciso o generico (nel caso di ossicle, pellicles, potion o sperme) o decisamente sbagliato (nerue). Alcuni problemi di equivalenza semantica vengono risolti - almeno apparentemente mediante il ricorso a sequenze sinonimiche costituite da due o anche tre elementi:

adustion, burning, or rosting; contusion, bruised, or beaten; crassitude, fatnesse or thicknesse; distended, stretched out, or out of ioynt; inspire, breath or blow into; laxatiue, loose, purging; lethall, mortall, deadly; $\$$ maniacque, mad: braine sick; medicine, remedie, or cure; obstruction, stopping, repressing; pest, the plague, or pestilence; pestiferous, contagious, hurtfull; pinguiditie, fatnes, or greatnesse; sanitie, health, or soundness; vlcer, bile, ${ }^{19}$ or botch.

$\$$ amorte, dead, extinguished, without life; crude, raw, not ripe, not digested; dolor, griefe, sorrow, or paine; languishing, pining, consuming, wearing away with griefe or sickness; vehement, earnest, strong, forcible.

Ovviamente, non rientrano in questa categoria quei casi in cui la sequenza di equivalenti del tecnicismo serve a renderne la polisemia: ad esempio, la voce «sanguine, bloudy, or of the colour of bloud» include sia un'accezione medica sia una di carattere generale; e il medesimo tipo di distinzione va fatto per «pregnant, wittie, substantiall, with child» e «vapor, moisture, ayre, hote breath, or reaking»: le accezioni mediche - «with child» e «hot breath» - si accostano agli usi del linguaggio comune.

La tecnica lessicografica di Cawdrey si rivela essere più efficace quando le definizioni manifestano chiaramente che il termine tecnico presentato va ascritto al linguaggio della medicina. Ciò avviene innanzitutto nei casi in cui le definizioni includono parole-chiave del discorso medico quali disease o sicknes (come già esemplificato per emerods, epilepsis e lethargie, a cui si può aggiungere «fluxe, disease of scouring»), ma anche sore («putrifie, to ware rotten, or corrupted as a sore»; «\$scarifie, to launce, or open a sore»; «vlcerate, to blister, or make full of sores»), wound («incision, cutting, in searching of a wound»; «incura-

19 Questa è una possibile grafia seicentesca per boil nel senso di 'pustola', non è bile nel senso di 'bile'. 
ble, past cure, a wound that cannot be healed») e phisicke, sia nel significato più ampio e nobile di 'scienza medica' («aphorisme, (g) generall rule in phisick»), sia in quello più contingente e umile di 'purgante' («decoction, liquor, wherein things are sod for phisicke»). ${ }^{20}$ Un'altra parola di chiara pertinenza medica è bumor che $A$ table alphabeticall include in definizioni che talvolta rimandano direttamente alla teoria degli umori della tradizione ippocratico-galenica («choller, (gr) a humour causing anger»; «melancholie, (g) black choler, a humor of solitarines, or sadnes»), talaltra indicano più genericamente i fluidi corporei («catharre, a flowing of humors from the head» e il già citato rbeu$m e$ ). Infine, gli utenti del dizionario possono essere aiutati a capire il significato del termine tecnico, o almeno a collocarlo nell'ambito del discorso medico, quando le definizioni includono riferimenti al lessico di base del corpo umano: agli esempi già elencati di gargarise, genitalles e nerue si possono aggiungere «\$ entrals, inward parts, as hart, liuer, \&c.», «beckticke, (g) inflaming the hart, and soundest parts of the bodie», «ligament, the string tying the bones together» e «ventricle, the stomacke which receiues the meate».

Restano escluse dalla tassonomia proposta alcune voci che sono comunque riconducibili all'ambito medico: non servivano di certo particolari competenze per riconoscere l'attinenza al discorso medico di voci quali «\$abortiue, borne before the time», «antidote, (g) a counterpoise, or remedy against poyson»o «contagious, that which corrupteth, or infecteth».

Altri esempi meritevoli d'attenzione sono la coppia quartane e tertian, e poi cordiall e preseruative. Infatti, i primi due termini - che Cawdrey presenta come «quartane, belonging to, or comming euery fourth day» e «tertian, belonging to euery third day» - hanno definizioni che di per sé non rimandano affatto all'ambito medico, ma in realtà sempre e solo in tale ambito vengono usati, a qualificare quei particolari tipi di febbre che si ripresentano ogni quattro o tre giorni. Le definizioni di «cordiall, comforting the hart» e «preseruative, that which defendeth» non rimandano immediatamente al discorso medico-scientifico, fanno piuttosto pensare ad altri contesti d'uso. Tuttavia, la ricerca lessicologica e semantica racchiusa nell'Oxford English Dictionary ricorda

${ }^{20}$ La polisemia di questo termine è evidente nella stessa definizione che ne dà Cawdrey: «phisicke, (g) medicine, helping, or curing». 
che, fin dal medioevo, l'aggettivo cordiall qualificava medicine, cibi o bevande da assumere per rinvigorire il cuore; e che preservative, secondo una definizione dell'OED, è «A medicine or other agent that gives protection from disease or infection». ${ }^{21}$

Di tali usi specifici Cawdrey non fa menzione; ciò non sorprende, se si riconsiderano i molti termini medici sopra elencati che il lessicografo definisce mediante il ricorso a uno o più sinonimi. Per limitare il commento a solo due degli esempi menzionati, le voci orifice e obstruction di $A$ table alphabeticall non accennano all'accezione medica dei due termini, che dovrà eventualmente essere dedotta da un reale contesto d'uso; ma basta spostarsi dal dizionario di Cawdrey a quello immediatamente successivo - An English expositor di John Bullokar - per notare un diverso approccio e l'evidente differenza, pur nella semplicità delle definizioni:

Cawdrey - orifice, mouth.

Bullokar - Orifice. The mouth of any thing, or the outward hole of a wound.

Cawdrey - obstruction, stopping, repressing.

Bullokar - Obstruction. A stopping, commonly in the bodie.

La distanza di An English expositor da A table alphabeticall si fa ancora più evidente in altri casi, come ad esempio i seguenti:

Cawdrey - anatomie, (g) cutting vp of the body.

Bullokar - Anatomie. An incision or cutting. The art of knowing the situation, office, and nature of all the parts of mans body.

Cawdrey - epilepsis, (g) the falling sicknes.

Bullokar - Epilepsie. The falling sicknesse, whereto most commonly children and yong folk are subiect. This disease is caused by some humor or vapor, suddenly stopping the passage of spirits in the braine, which the brain striuing to expel, causeth the patient to fall downe, and commonly fome at the mouth.

21 Oxford English Dictionary (OED) Online, http://www.oed.com, s.v. cordial e preservative. 
Cawdrey - phlebotomie, (g) letting bloud.

Bullokar - Pblebotomie. Letting of blood. Phisitions (as is written) learned this practise first of a beast called Hyppopotamus, liuing in the riuer Nilus; which being of a rauenous nature, and therefore often ouercharged with much eating, is wont to seeke in the banks, for some sharpe stub of a Reede; vpon which pricking his leg, he thereby easeth his full body, stopping the bleeding afterward with mud.

Il differente trattamento lessicografico di queste voci si spiega in due modi: innanzitutto, come è stato ricordato nella sezione 2, John Bullokar è medico, e quindi la sua presentazione è chiaramente influenzata dalle sue competenze e interessi professionali ${ }^{22}$ in secondo luogo, mentre Cawdrey si ispira alla pratica lessicografica dei glossari con le loro sintetiche presentazioni, Bullokar opta spesso per definizioni di carattere enciclopedico più che strettamente lessicografico.

\section{Considerazioni ConClusive}

Il confronto, qui solo accennato fra il dizionario di Cawdrey e quello di Bullokar, così come il richiamo sopra delineato alle fonti usate da Cawdrey, possono suggerire alcune brevi considerazioni conclusive sul posizionamento linguistico e culturale di $A$ table alphabeticall nella genesi della lessicografia monolingue e, parallelamente, della terminologia scientifica in Inghilterra.

Robert Cawdrey è ricordato come l'iniziatore della lessicografia inglese monolingue, e questo è un merito che gli va riconosciuto. Tuttavia, se lo scopo primario della lessicografia bilingue e plurilingue è quello di stabilire una relazione o corrispondenza semantica tra forme equivalenti di lingue diverse, e se lo scopo primario della lessicografia monolingue è invece quello di definire il significato - ovvero, delimitare l'area semantica - delle parole di una data lingua, allora dif-

22 McConchie, What were Robert Cawdrey's hard words? Learned terms and A table alphabeticall (1604), 185, specifica: «Bullokar's work contains approximately 4000 entries, of which 318 (7,95\%) are medical in the sense that they either have a clear field-marker (52) or that they are clearly medical by Bullokar's own definition. This is certainly higher than in the case of Cawdrey». 
ficilmente si potrà sfuggire alla conclusione che la novità rappresentata da Cawdrey esiste più nella forma che nella sostanza. Formalmente, il suo è un dizionario monolingue; sostanzialmente, il suo è, per così dire, un bilingue inglese-inglese, in cui non sono poste a confronto due lingue diverse, ma due registri stilistici diversi - uno alto e dotto, l'altro basso e colloquiale - della stessa lingua; in ogni caso, Cawdrey riesce ben poco a definire le «hard vsuall English wordes» su cui concentra il suo interesse, e spesso si rivelano inefficaci le «plaine English words, gathered for the benefit $\&$ helpe of Ladies, Gentlewomen, or any other vnskilfull persons» che egli propone.

Anche dal punto di vista della nascente terminologia scientifica in lingua inglese, che nel corso del Seicento verrà ampiamente a sostituire quella latina, il contributo di Cawdrey è limitato, come si è potuto verificare analizzando le voci d'argomento medico: espliciti riferimenti alla scienza medica sono rari; anche quando chi consultava il dizionario scopriva che un dato termine era di carattere medico, l'informazione offerta appariva solitamente limitata e non sufficientemente precisa. Inoltre, pur riconoscendo i limiti che un dizionario di sole 2500 voci può legittimamente avere, non si coglie alcun intento di sistematicità nella selezione del lemmario operata da Cawdrey: per fare un solo esempio, il dizionario elenca le voci asperation (refuso per aspiration) e respiration, ma non inspiration, che è sostituita dal verbo «inspire, breath or blow into».

Considerato nella prospettiva della storia della lessicografia inglese, si può dunque forse paradossalmente concludere che il primo dizionario inglese monolingue vada correttamente giudicato nella prospettiva della continuità e dell'influsso esercitato dalla tradizione precedente piuttosto che in quella del cambiamento e dell'innovazione. Tuttavia, al di là dei risultati concreti, a Cawdrey e alla sua $A$ table alphabeticall va riconosciuto il merito di avere focalizzato l'attenzione sui termini dotti di derivazione straniera, e di avere quindi avviato la tradizione degli hard-word dictionaries che nel suo complesso - al di là dei pregi e dei difetti delle singole opere - ha aperto la strada alla compilazione dei dizionari tecnici e delle enciclopedie nel primo Settecento.

All'inizio del Seicento la lessicografia inglese non è ancora pronta a raccogliere e correttamente codificare termini e concetti che la scienza moderna stava elaborando e assimilando; solo facendosi via via più salde e mature, le acquisizioni medico-scientifiche del tempo potevano 
trasformarsi nei 'farmaci a piccole dosi' rappresentati dalle voci delle opere lessicografiche ed enciclopediche compilate e pubblicate per divulgare competenze linguistiche e saperi scientifici al di fuori della ristretta cerchia dei dotti, a favore delle classi sociali istruite ma prive di conoscenze specialistiche e desiderose di apprendere. 